\title{
Single ice crystal measurements during nucleation experiments with the depolarization detector IODE
}

\author{
M. Nicolet ${ }^{1}$, O. Stetzer ${ }^{1}$, F. Lüönd ${ }^{1}$, O. Möhler ${ }^{2}$, and U. Lohmann ${ }^{1}$ \\ ${ }^{1}$ Institute for Atmospheric and Climate Science, ETH Zurich, 8092 Zurich, Switzerland \\ ${ }^{2}$ Institute for Meteorology and Climate Research, Forschungszentrum Karlsruhe, Germany \\ Received: 11 September 2008 - Published in Atmos. Chem. Phys. Discuss.: 16 December 2008 \\ Revised: 27 November 2009 - Accepted: 11 December 2009 - Published: 18 January 2010
}

\begin{abstract}
In order to determine the efficiency of different aerosol particles to nucleate ice, an Ice Optical DEpolarization detector (IODE) was developed to distinguish between water droplets and ice crystals in ice nucleation chambers. A laser beam polarized linearly (power: $50 \mathrm{~mW}$, wavelength: $407 \mathrm{~nm}$ ) is directed through the chamber. The scattered light intensity from particles is measured at a scattering angle of $\Theta=175^{\circ}$ in both polarization components (parallel and perpendicular). The ratio between the perpendicular intensity over the total one yields the depolarization ratio $\delta$. Single particle detection is possible, using a peak detection algorithm. For high particle concentrations, a real-time signal averaging method can also be run simultaneously.

The IODE detector was used in connection with the Zurich ice nucleation chamber during the ICIS 2007 workshop where ice nucleation experiments were performed with several aerosol types. In presence of ice crystals, a depolarization ratio could be measured on a particle-by-particle basis. Mean values of $\delta$ ranged from 0.24 to 0.37 and agree well with theoretical calculations.
\end{abstract}

\section{Introduction}

The role of aerosol particles in droplet activation has been recognized for almost 120 years as an essential component of cloud formation (Spurny, 2000). These particles are called cloud condensation nuclei (CCN). Increases in $\mathrm{CCN}$ due to anthropogenic activity also affect climate by changing the cloud characteristics (Lohmann and Feichter, 2005). Both the increase in cloud lifetime due to more and smaller cloud droplets and the associated radiative transfer changes are contributing to the indirect aerosol effect in climate forcing.

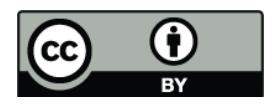

Correspondence to: O. Stetzer

(olaf.stetzer@env.ethz.ch)
According to the last IPCC report, the cloud albedo effect amounts to a global mean forcing of $-0.7 \mathrm{~W} \mathrm{~m}^{-2}(-1.8$ to $-0.3 \mathrm{~W} \mathrm{~m}^{-2}$ ), but the level of scientific understanding is still low (Forster et al., 2007). The aerosol properties affecting their ability to act as CCN are rather well understood. However, there is a lack of knowledge of the ice nucleation capabilities of aerosol particles (Cantrell and Heymsfield, 2005; Szyrmer and Zawadzki, 1997). The theory for heterogeneous nucleation including the molecular nature of its initiation and growth stages is not fully understood. The ice formation efficiency as a function of aerosol particle properties is poorly understood because of the variety of heterogeneous ice nucleation modes. Four nucleation modes are typically distinguished (Vali, 1985). We focus on the deposition and immersion modes: Deposition freezing occurs when water vapor deposits directly onto the aerosol particle, forming ice without any transition through the liquid phase in a supersaturated environment with respect to ice. Ice nuclei (IN) can also initiate freezing from within a cloud droplet by immersion freezing where the particle is suspended in the interior of the water droplet. Another important unresolved problem is to understand the link between ice nucleation and cloud properties that affect climate. Therefore, a greater understanding of the ice nucleation mechanisms is required to improve their representation in climate models. One good approach is to perform measurements of ice nucleation processes in the laboratory under controlled conditions. This can be achieved with cloud diffusion chambers.

The Zurich Ice Nucleation Chamber (ZINC) (Stetzer et al., 2008) allows activation and growth of IN by vapor deposition to detectable ice crystal sizes (from $1 \mu \mathrm{m}$ in diameter) in an ice supersaturated environment. The design of the chamber is based on the successful Colorado State University (CSU) instrument (Rogers, 1988, 1993) but it uses two parallel walls instead of two concentric cylinders. The ZINC chamber also has an evaporation section located in the lower part of the chamber. As both walls are set to the same temperature in

Published by Copernicus Publications on behalf of the European Geosciences Union. 
this section, it is saturated with respect to ice and thus subsaturated with respect to water, allowing water droplets to evaporate before detection. One of the limitations of the existing continuous flow diffusion chambers (CFDC) is the indirect detection of ice crystals by particle size (Rogers et al., 2001). If higher supersaturations and warmer temperatures are investigated, immersion freezing can take place as well. In this case, we may have both the liquid and the solid phase present during experiments so that we have to be able to differentiate between them. If water droplets and ice crystals are present simultaneously, it is not possible to distinguish between them based on their size. To overcome this limitation, a new detection system was built to distinguish between droplets and ice crystals. The property of light depolarization is used, which considers that ice particles are non-spherical and thus induce depolarization of the light whereas water droplets do not (Liou and Lahore, 1974). This device follows the design that is used at the AIDA Chamber (Büttner, 2004). Whereas Büttner (2004) measures average signals of a collection of particles, we target to detect single particles as IN concentrations in the atmosphere are typically very low $\left(\sim 10 \ell^{-1}\right.$ at $\left.-25^{\circ} \mathrm{C}\right)$ (Gőtz et al., 1991). Several experimental applications have been built for single particle detection such as the Small Ice detector (SID) (Hirst et al., 2001), the Particle Measurement System forward scattering probe (FSSP) (Lawson and Cormack, 1995) and the Video Ice Particle Sampler (VIPS) (McFarquhar et al., 2002). Other optical systems dealt with ice nucleation of single levitated (Krämer et al., 1996) or free-falling droplets (Wood et al., 2002) where detection of ice particles using depolarization has been performed. The influence of the particle orientation on the depolarization ratio is large, according to a previous modeling study (Nicolet et al., 2007). The single particle detection aspect has a major influence in terms of scattered light intensity and amount of light depolarization expected in that case.

In the present work, the design and the software of the ice detector device called IODE (Ice Optical DEtector) will be presented. The IODE device was connected to the bottom of the ZINC chamber where the scattering and polarization of water droplets and ice crystals were investigated. The results obtained with this detector will be discussed in the following chapters, along with perspectives, challenges and possible future work.

\section{Theory}

The depolarization technique is commonly used in remote sensing applications, such as lidars. It has been proposed that depolarization can be used as a parameter for distinguishing between ice crystals and water droplets (Liou and Schottland, 1971). Liquid water particles are assumed to be spherical and therefore cause no depolarization whereas ice crystals can be considered as being non-spherical, causing partial depolar- ization of the scattered light in the backward direction (Liou and Lahore, 1974).

In the 1960s, early atmospheric tests of the lidar technology were aimed at assessing the information content of the backscattering depolarization when probing clouds (Sassen, 1995). Thus, it was indicated that lidars using a polarized light source operating in the visible and near infrared wavelengths of the electromagnetic spectrum should be capable of differentiating between water droplets and ice particles. To explain this property in a purely theoretical way, we use the so-called Stokes parameters given in a $4 \times 1$ vector to describe the properties of the laser source and the scattered light:

$\mathbf{I}=\left[\begin{array}{c}I \\ Q \\ U \\ V\end{array}\right]$

where $\mathbf{I}$ is the net monochromatic energy flux. $Q$ and $U$ express the state of linear polarization and $V$ describes the state of circular polarization. The Stokes parameters are always defined with respect to a reference plane. Defining the scattering plane with reference to the fully linearly polarized laser beam, the Stokes vector of the incident laser source $\mathbf{I}^{\text {inc }}$ becomes $[1,1,0,0]^{T}$. It is then possible to define the $4 \times 4$ scattering matrix $\mathbf{F}$ to explicit the Stokes parameters of the scattered beam as a function of the incident Stokes parameters. This definition is given with respect to the scattering plane

$\mathbf{I}^{\mathrm{sca}}=\frac{C_{\mathrm{sca}}}{4 \pi r^{2}} \mathbf{F}(\Theta) \mathbf{I}^{\mathrm{inc}}$

where $C_{\text {sca }}$ is the average particle scattering cross section, $r$ is the distance between the particle and the detector and $\Theta$ is the scattering angle. The concept of the scattering matrix is particularly useful when all orientations of the particles are of equal probability, e.g. particles are randomly oriented. If each particle has a plane of symmetry and/or particles and their mirror particles are present in equal number, such a scattering medium is called macroscopically isotropic and symmetric. Due to the symmetry, the scattering matrix $\mathbf{F}$ for such a media is invariant with respect to the choice of the scattering plane. The average scattering cross section is independent of the direction and polarization of the incident beam and the scattering matrix for this media has a block-diagonal structure (van de Hulst, 1957):

$\mathbf{F}(\Theta)=\left[\begin{array}{cccc}F_{11}(\Theta) & F_{12}(\Theta) & 0 & 0 \\ F_{12}(\Theta) & F_{22}(\Theta) & 0 & 0 \\ 0 & 0 & F_{33}(\Theta) & F_{34}(\Theta) \\ 0 & 0 & -F_{34}(\Theta) & F_{44}(\Theta)\end{array}\right]$ 
The representation of the scattering matrix considering exact backscattering direction $\left(\Theta=180^{\circ}\right)$ from the polarized light source is then given by (Mishchenko and Hovenier, 1995):

$\mathbf{F}\left(\Theta=180^{\circ}\right)=\left[\begin{array}{cccc}F_{11} & 0 & 0 & 0 \\ 0 & F_{22} & 0 & 0 \\ 0 & 0 & F_{33} & 0 \\ 0 & 0 & 0 & F_{44}\end{array}\right]$

The ratio of the perpendicular to the parallel polarization components of backscattered light is defined by the linear depolarization ratio $\delta$ and is equal to:

$\delta=\frac{I_{\perp}^{\text {sca }}}{I_{\|}^{\text {sca }}}=\frac{F_{11}\left(180^{\circ}\right)-F_{22}\left(180^{\circ}\right)}{F_{11}\left(180^{\circ}\right)+F_{22}\left(180^{\circ}\right)}$

For spheres, $F_{11}\left(180^{\circ}\right)=F_{22}\left(180^{\circ}\right)$ which leads to the finding of $\delta=0$ for scattering by water droplets.

The depolarization technique was also used for in situ phase discrimination in the AIDA (Aerosol Interaction and Dynamics in the Atmosphere) chamber where ice nucleation experiments were performed (Möhler et al., 2003). The detection device uses an $\mathrm{Ar}^{+}$-laser $(\lambda=488 \mathrm{~nm})$ linearly polarized and directed horizontally through the chamber. Scattering intensities are measured with photomultipliers (PMT) in the forward direction $\left(\Theta=4^{\circ}\right)$, and polarization resolved in the backward direction $\left(\Theta=176^{\circ}\right)$. The main difference to a Lidar is that perfect backscattering measurements are not performed due to technical reasons. In that case, $F_{12}$ and $F_{21}$ are non-zero elements of the scattering matrix $\mathbf{F}$ and $F_{12}=F_{21}$ with the assumption of a collection of randomly oriented particles. Therefore, the depolarization ratio is defined as the ratio between the perpendicular-polarized scattered light and the total one assuming an incident light polarized parallel to the scattering plane:

$\delta_{\|}=\frac{I_{\perp}^{\text {sca }}}{I_{\|}^{\text {sca }}+I_{\perp}^{\text {sca }}}=\frac{1}{2} \frac{F_{11}-F_{22}}{F_{11}-F_{12}}$

For our specific experiments, we have to consider two important aspects. First we are dealing with very low concentrations of aerosols particles in the ZINC chamber. Therefore, single detection events occur as opposed to collections of particles as discussed above. Secondly, as single particles have to be detected one by one, they are positioned in a specific orientation so that light scattering from a given orientation of the ice crystal has to be considered. This experimental definition of the depolarization ratio can be related to the theoretical one that can be derived from the elements of the phase matrix $\mathbf{Z}$. This $4 \times 4$ matrix is obtained from the phase matrix $\mathbf{S}$ that linearly transforms the electric field vector components of the incident wave into the electric field vector components of the scattered wave. $\mathbf{Z}$ takes the scattering angle and the orientation of the particle given by its Euler angles into account (Mishchenko, 2000). The Stokes vector of the scattered light can then be obtained as:

$\mathbf{I}^{\mathrm{sca}}=\frac{1}{r^{2}} \mathbf{Z I}^{\mathrm{inc}}$
With a laser beam polarized parallel to the scattering plane, we have $\mathbf{I}^{\text {inc }}=[1,1,0,0]^{T}$. Only the elements $Z_{11}, Z_{12}, Z_{21}$ and $Z_{22}$ can be considered and the Stokes vector of the scattered light can be written as:

$\left[\begin{array}{l}\mathbf{I}^{\text {sca }} \\ \mathbf{Q}^{\text {sca }}\end{array}\right]=\frac{1}{r^{2}}\left[\begin{array}{ll}Z_{11} & Z_{12} \\ Z_{21} & Z_{22}\end{array}\right]\left[\begin{array}{l}\mathbf{I}^{\text {inc }} \\ \mathbf{Q}^{\text {inc }}\end{array}\right]$

and

$\left\{\begin{array}{l}\mathbf{I}^{\mathrm{sca}}=I_{\|}^{\mathrm{sca}}+I_{\perp}^{\mathrm{sca}} \\ \mathbf{Q}^{\mathrm{sca}}=I_{\|}^{\mathrm{sca}}-I_{\perp}^{\text {sca }}\end{array}\right.$

Considering that $\mathbf{Q}^{\text {inc }}=\mathbf{I}^{\text {inc }}, \quad \mathbf{I}^{\text {sca }}=\left(Z_{11}+Z_{12}\right) \mathbf{I}^{\text {inc }}$ and $\mathbf{Q}^{\text {sca }}=\left(Z_{21}+Z_{22}\right) \mathbf{Q}^{\text {inc }}$, the following definition of the depolarization ratio of the light results from Eqs. (6) and (7):

$\delta_{\|}=\frac{I_{\perp}^{\mathrm{sca}}}{I^{\mathrm{sca}}}=\frac{Z_{11}+Z_{12}-Z_{21}+Z_{22}}{2\left(Z_{11}+Z_{12}\right)}$

Similarly, the Stokes vector is given as $\mathbf{I}^{\text {inc }}=[1,-1,0,0]^{T}$ when a light source polarized perpendicular to the scattering plane is used. Consequently, $\mathbf{Q}^{\text {inc }}=-\mathbf{I}^{\text {inc }}$ and the depolarization ratio can be defined as:

$\delta_{\perp}=\frac{I_{\|}^{\text {sca }}}{\mathbf{I}^{\text {sca }}}=\frac{Z_{11}-Z_{12}+Z_{21}-Z_{22}}{2\left(Z_{11}-Z_{12}\right)}$

Here $Z_{12} \neq Z_{21}$ as a specific orientation of a single particle is taken into account in this study. Using a collection of randomly oriented particles leads to $Z_{12}=Z_{21}$ and the same expression as in Eq. (6) is obtained.

\section{Description}

\subsection{Experimental apparatus}

The IODE detector was placed at the lower end of the evaporation section of ZINC in its standard configuration (Stetzer et al., 2008). Although droplets only reach the detector at very high supersaturations this configuration was neccesary to obtain data for both the IODE detector and the standard OPC for comparison of the data. In the IODE setup, the laser light source is a linearly polarized (100:1) $50 \mathrm{~mW} \mathrm{GaN} \mathrm{diode}$ laser operating at $407 \mathrm{~nm}$ (Oxxius OXV-405). Collimation is already included in the laser so that the beam divergence remains under $1 \mathrm{mrad}$. The laser beam is tilted downwards with an angle of $5^{\circ}$ with respect to the horizontal plane to have a scattering angle of $175^{\circ}$. The beam (Gaussian type) has an elliptical diameter of $1 \times 2.5 \mathrm{~mm}$ and the resulting overlap region (area resulting from the intersection between the laser beam and the detector field of view i.e. the area where the particles can be detected) has a volume of $302 \mathrm{~mm}^{3}$. The whole detection system is shown in Fig. 1.

In the chamber, the particles sediment in a laminar flow (as confirmed by FLUENT simulations) so that turbulence does not have to be taken into account. As the particles 


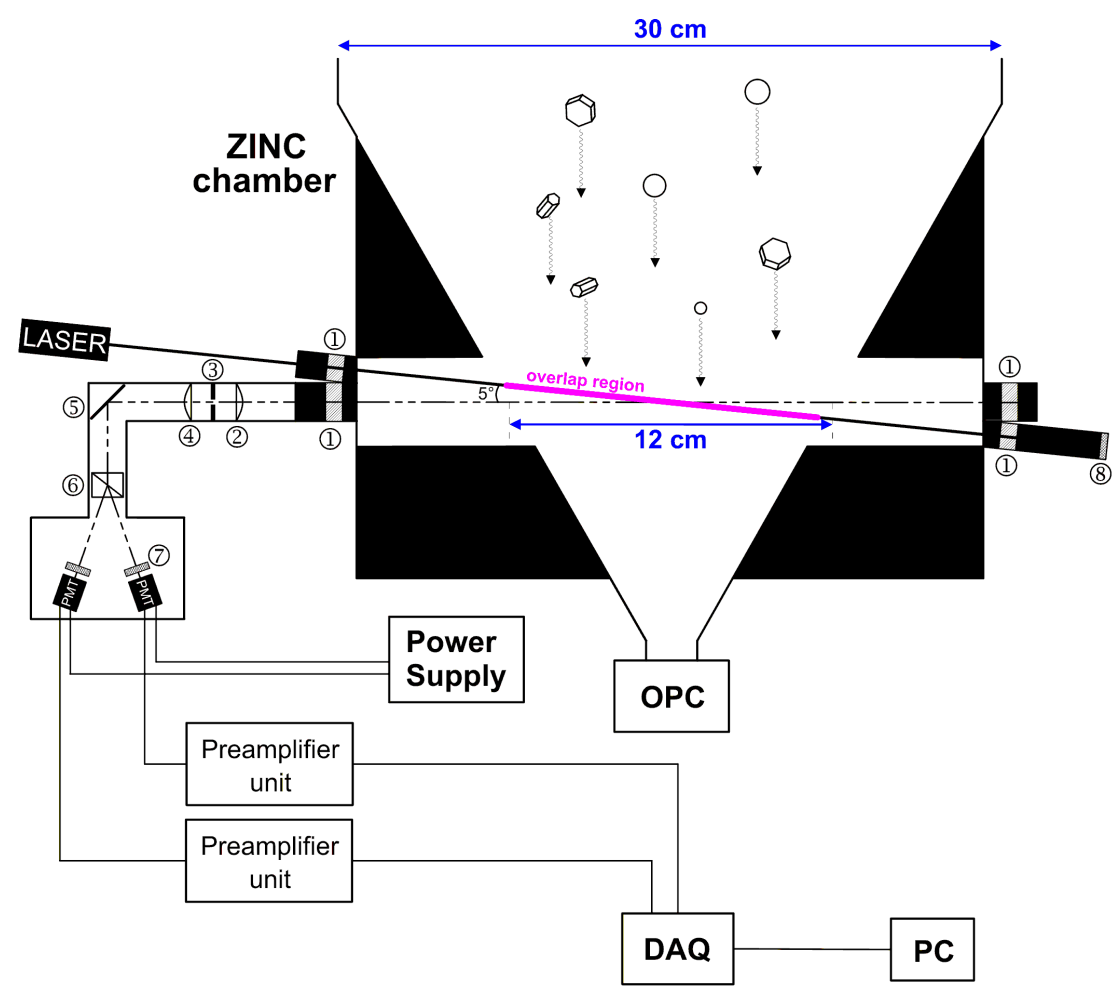

(1) coated windows
(2) convergent lens $(f=25 \mathrm{~mm})$
(3) pinhole
(4) convergent lens $(f=19 \mathrm{~mm})$

(5) mirror

(6) Wollaston prism

(7) bandpass filter

(8) beam dumper

Fig. 1. Schematic diagram of the experimental setup.

may be randomly distributed over the width of the chamber, they have to be detected at any location along the overlap region. Coated windows are mounted on both apertures to keep the chamber tight during experiments. Therefore, all optical components are separated from the chamber. The scattering plane is vertical in this configuration and the polarization can be easily switched in parallel or perpendicular position by turning the laser by $90^{\circ}$ to the cited plane in order to get $\delta_{\|}$ or $\delta_{\perp}$, respectively.

Two convergent lenses of focal length 19 and $25 \mathrm{~mm}$ and a pinhole form a telescope to collect the scattered light and to prevent parasite light from the environment to enter the system. After being reflected at $90^{\circ}$ with a coated mirror, a Wollaston prism then splits the beam into its parallel and perpendicular polarization components. It has the specificity over other polarizing prism types of splitting the scattered ray almost symmetrically. One advantage is to avoid multiple reflections in the prism if the angles are not precisely adjusted. The angle between the two resulting beams is $\sim 16^{\circ}$. Before detection by two Hamamatsu H5783P photomultipliers (PMT), both rays pass through bandpass filters that stop wavelengths different from $407 \mathrm{~nm}$ to avoid diffused light from the environment reaching the photocathodes of the PMTs. The amplification gain $g$ of the PMTs can be regulated from $2.81 \times 10^{2}$ to $2.3 \times 10^{6}$. A Hamamatsu C7319 preamplifier unit placed directly at the signal output of each PMT transforms and amplifies the incoming current signal into a voltage signal. It has three switchable conversion ratios $\left(R_{\text {conv }}\right) ; 10^{5}, 10^{6}$ and $10^{7}$ V/A and also has two switchable bandwidth selections; 20 and $200 \mathrm{kHz}$. The complete transformation from the voltage signal to physical units can be obtained with the following formula:

$I[\mathrm{pW}]=\frac{U[\mathrm{~V}] \times 10^{15}}{\xi[\mathrm{mA} / \mathrm{W}] \times g \times R_{\mathrm{conv}}[\mathrm{V} / \mathrm{A}]}$

where $\xi$ is the cathode radiant sensitivity, $g$ the amplification gain of the PMT and $R_{\text {conv }}$ the conversion ratio of the amplifier. As the signal intensities might be very low due to single particle scattering, the challenge is to reduce the background signal and its standard deviation as much as possible to have the best possible detection accuracy. The background 
in both channels is included in the experimental definition of the depolarization ratios that can be used in our device:

$\delta_{\|}=\frac{I_{\perp}^{\mathrm{sca}}-I_{\perp}^{\mathrm{BG}}}{\left(I_{\perp}^{\mathrm{sca}}-I_{\perp}^{\mathrm{BG}}\right)+\left(I_{\|}^{\mathrm{sca}}-I_{\|}^{\mathrm{BG}}\right)}$

$\delta_{\perp}=\frac{I_{\|}^{\mathrm{sca}}-I_{\|}^{\mathrm{BG}}}{\left(I_{\perp}^{\mathrm{sca}}-I_{\perp}^{\mathrm{BG}}\right)+\left(I_{\|}^{\mathrm{sca}}-I_{\|}^{\mathrm{BG}}\right)}$

where $I_{\|}^{\mathrm{BG}}$ and $I_{\perp}^{\mathrm{BG}}$ stand for the average background intensities in the parallel and perpendicular channels (with respect to the scattering plane). Background measurements are done before activation experiments and take into account the scattered light from aerosols, air molecules and reflections from the walls.

\subsection{Data acquisition}

To measure and analyze the signal at the preamplifier outputs, a 4-channel analog input data acquisition unit (DAQ) is used to provide 16-bit simultaneous sampling with integrated signal conditioning (National Instrument, 9215 module). It is connected to a PC with a USB interface (National Instrument, USB-9162 USB carrier). The maximum sampling rate is $100 \mathrm{kHz}$ per channel and the typical operating voltage range is $10.4 \mathrm{~V}$. According to simulations made by Stetzer et al. (2008) with the FLUENT computational fluid dynamics software, the average velocity of particles inside the ZINC chamber is $0.1 \mathrm{~m} / \mathrm{s}$. It rises up to $0.4 \mathrm{~m} / \mathrm{s}$ at the height of the detector windows due to the shrinking in the collector cone. As the laser beam has a diameter of $2.5 \mathrm{~mm}$ in its parallel configuration, the interaction time between the beam and a particle is $\sim 6 \mathrm{~ms}$. With a given sampling rate of $10 \mathrm{kHz}$, one particle will then be sampled with approximately 60 points. For the perpendicular setup, the beam diameter is $1 \mathrm{~mm}$, leading to an interaction time of $2.4 \mathrm{~ms}$. The signal peak is theoretically defined with 24 points which is still sufficient for a good peak detection.

\subsection{Data analysis}

A program designed with LabView (National Instrument) was made for real-time data analysis and includes a peak detection algorithm for particle detection and depolarization measurements. The peak detection algorithm scans the signal from the photomultipliers and searches for peaks in the following way: A peak is considered valid if it has the following characteristics: First, the elements of a sequence of points begins below a threshold limit $\tau$, then exceeds it for some time, and then returns to a value below $\tau$. Second, the interval that exceeds $\tau$ is greater than or equal to a given width $w$. The peak-finding algorithm then fits a parabolic function to the data points above $\tau$. Using a least-squares algorithm, the peak detection algorithm finally returns amplitudes (intensities) and locations (time) of the peaks.
The determination of the best value for the threshold limit $\tau$ is done by the analysis of the background signal. If we assume that the probability density function for the background intensity follows a normal distribution, then the threshold value $\tau$ for each channel $\left(\tau_{\|}\right.$and $\tau_{\perp}$ ) can be determined with the application of the statistical law:

$\tau=\mu_{\mathrm{BG}}+n \sigma_{\mathrm{BG}}$

Where $n$ is a positive number. To determine the depolarization ratio in the backward direction, both peaks corresponding to the parallel and perpendicular channel have to appear at the same time, considering a tolerance time interval $\Delta t$ that should be set within an optimum value. Peak detection is made first in the parallel channel. If a peak in the perpendicular channel is then located at the same time $\pm \Delta t, \delta$ can be obtained from both peak intensities using Eqs. (13) and (14). If no corresponding peak in the perpendicular channel is found, $\delta$ is set to zero.

For data filtering, a Lowpass filter is used to suppress noise while keeping peak characteristics in terms of intensity, location and width. Moreover, the standard deviation of the background is lowered and therefore the threshold limit $\tau$. Hence, the detection efficiency for small particles increases.

\subsection{Experiments}

Ice nucleation experiments discussed here were carried out during the International Workshop on Comparing Ice Nucleation Measuring systems (ICIS 2007) that was held in Karlsruhe, Germany. The main goal of this workshop was to compare different ice nucleation devices such as the ZINC chamber (Stetzer et al., 2008), the FINCH chamber (Bundke et al., 2008) and the CSU chamber (Rogers, 1988) during ice nucleation experiments. Several aerosol types were used, like Arizona test dust, soot particles, Canary Islands dust (CID), Israel dust (ID), Saharan dust (SD), and Snomax bacteria (SB) (Morris et al., 2004). This latter product stems from cells of $P$. syringae bacteria that have been freeze-dried and then killed by gamma radiation. The aerosol particles were sampled mostly from the NAUA chamber, which is part of the AIDA facility. It is a small-sized $\left(3.7 \mathrm{~m}^{3}\right)$ evacuable aerosol chamber made of stainless steel operating at ambient temperature (stability: $\pm 2 \mathrm{~K}$ ) (Schnaiter et al., 2006). All aerosols were generated and made available in the NAUA chamber.

Experiments with the ZINC chamber were conducted in the following way: Activation sequences started by decreasing the temperature of the cold wall and setting the warm wall to a constant temperature, leading to an increase of the supersaturation inside the chamber. Each activation cycle was realized with a different warm wall temperature such that every activation occurred at a different temperature. The aerosol flowrate was fixed at $0.2 \mathrm{lpm}$ for most experiments, as first tests with Arizona test dust showed that standard flow of $1 \mathrm{lpm}$ resulted in too high aerosol concentrations within 


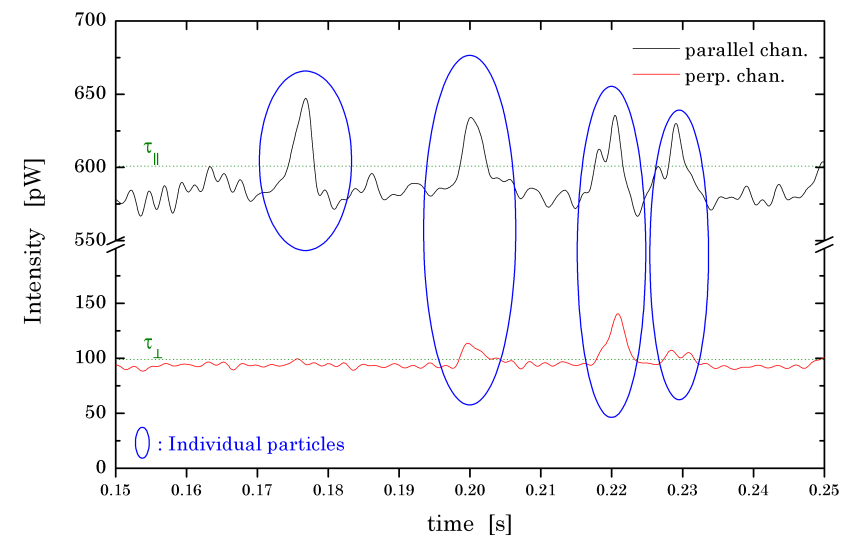

Fig. 2. 100-ms duration real time measurements of the 4th activation experiment made with Snomax bacteria.

the ZINC chamber. This could lead to coincidence problems with the optical particle detectors. The sheath air flow rates were both set to $2 \times 4.9 \mathrm{lpm}$ accordingly to maintain a total flow of $10 \mathrm{lpm}$ through the instrument. During all experiments, a 500-Hz lowpass filter was applied to the IODE raw data. The conversion rate was set to $10^{7} \mathrm{~V} / \mathrm{A}$ for both. The Preamplifier bandwidth was set to $20 \mathrm{kHz}$. This frequency is sufficient as the software samples the data with a rate of $10 \mathrm{kHz}$. The width $w$ for peak detection was set to 15 and the parameter $n$ to 3 for the threshold determination for both channels.

All peaks in the parallel and perpendicular channel were recorded during the activation cycles. The depolarization ratios were then directly derived from the peak intensities from the software. In addition to the IODE detector, an optical particle counter (OPC: Climet CI 3100) was connected to the bottom of the chamber to count the ice particles in the traditional way. This is done by amplifying and directing the signal of the OPC to a multichannel analyzer (AmpTek, Pocket MCA 8000A) to classify the ice crystals by size (Stetzer et al., 2008). However, the optical particle counter is not able to provide any information about the phase of the particles detected. Finally, a condensation particle counter (CPC) was used to obtain the total aerosol concentration. The OPC activated fraction is then determined with the CPC data.

\section{Results}

\subsection{Snomax bacteria}

For the Snomax bacteria experiment, A part of a 1.5-s duration real-time signal during the 4th activation experiment is first shown in Fig. 2 as an example where both parallel and perpendicular channels are mentioned.

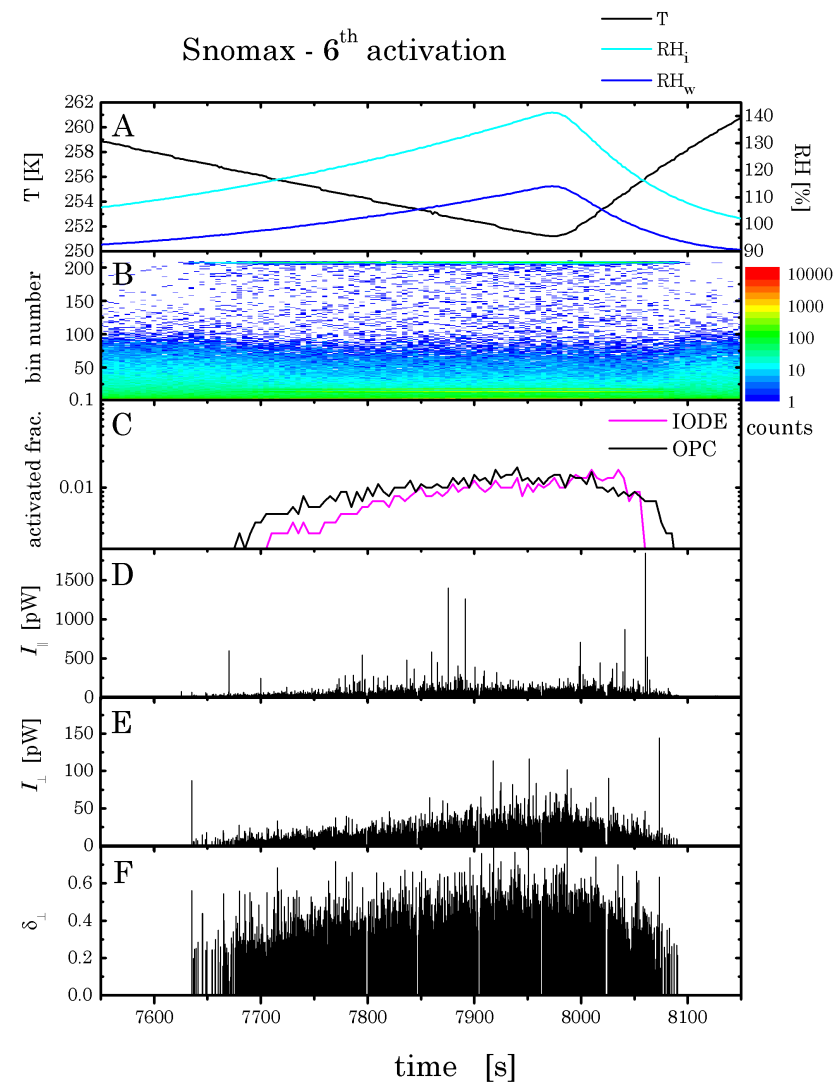

Fig. 3. Scheme of a typical activation experiment (Snomax bacteria 6th activation). Panel (A) shows the temperature and humidity profiles calculated from the wall temperatures and flows during the experiment. Panel (B) illustrates the raw data from the optical particle counter as a color coded intensity spectra. The activated fraction of aerosol particles calculated from the OPC and IODE datasets are plotted in panel (C). The parallel and perpendicular intensities of the IODE detector are given in panels (D) and (E). Panel (F) illustrates the depolarization ratio obtained from both intensities.

One typical activation event (Snomax 6th activation experiment) is described in Fig. 3. The temperature of the warm wall was set to $263 \mathrm{~K}$.

Figure 3a shows the temperature $T$ and humidity profiles with respect to ice $\mathrm{RH}_{i}$ and to water $\mathrm{RH}_{w}$ for the sample flow. The humidities are calculated by using the parametrizations from Murphy and Koop (2005). Figure 3b illustrates the OPC raw data as a color-coded size intensity plot. Individual size distributions are obtained every $5 \mathrm{~s}$. The activated fraction derived from the OPC data is shown in Fig. 3c. The number of activated particles is obtained as following: on one hand, the aerosol concentration is obtained directly from the NAUA chamber from the condensation particle counter. On the other hand, the number of activated particles is calculated by integrating the OPC dataset approximately from channel 90 to 160 upward, depending on the aerosol size 
distribution. As channel 90 corresponds to a particle diameter of approximately $2.5 \mu \mathrm{m}$ in diameter according to our calibration, particles of this range of size are considered as ice particles. Channel 160 is related to a diameter of around $4 \mu \mathrm{m}$. The ratio of these two numbers is then the activated fraction. Figure $3 \mathrm{~d}$ and e shows the individual peak intensities from the parallel and the perpendicular channel obtained with the IODE detector, respectively. Finally, Fig. $3 f$ depicts the depolarization ratio obtained from the intensities as discussed above.

Snomax bacteria activate around water activation. As this is within the regime of immersion freezing, we may have ice crystals and water droplets simultaneously. Due to the evaporation section in the lower part of the ZINC chamber, these water droplets formed in the upper part should evaporate and we should only have ice particles detected by the OPC and the IODE detector. However, if the relative humidity with respect to water is above a certain critical value, the water droplets do not have sufficient time to evaporate entirely due to their large sizes. Therefore, they are then detected with both detectors. We call this a "water breakthrough" event where we expect detection of ice crystals and water droplets at the same time although the evaporation section of the chamber is always sub-saturated with respect to water. Beyond this limit, water droplets do not have sufficient time to evaporate completely and therefore ice and water may coexist at the level of detection. We made calculations for growth and evaporation of droplets for two sections with constant conditions to estimate the supersaturations above which droplets survive the evaporation section and reach the OPC. We find good agreement with the experimental breakthrough line for warm temperatures (250 and $255 \mathrm{~K})$. For colder temperatures, the calculated breakthrough is at higher supersaturations than measured. However, this deviation is very likely a consequence of the simple twosection approach without taking into account the transitional supersaturation between main chamber and evaporation section. This transition is longer and more pronounced at high supersaturations and therefore low temperatures and results in longer growth times before reaching sub-saturated conditions.

In the Snomax 6th activation experiment, the particle activation started at about $t=7630 \mathrm{~s}(T=257.5 \mathrm{~K}$ and $\mathrm{RH}_{i}=110.2 \%$ ). This is revealed in the OPC raw data (panel b) where ice crystals are detected (appearance of counts in channels from about 90 until 210). Everything before that time is considered as background (air loaded only with aerosols). Consequently, this is the background signal taken into account for the IODE peak algorithm tuning (determination of the threshold $\tau$ ). The start and the increase of the activated particles fraction (panel c) was also remarkable as the curve rose to 0.01 between roughly $t=7800$ to $8000 \mathrm{~s}$. The activation was indicated with peaks in both the parallel and perpendicular channels found by the IODE detector. The onset of ice in the IODE detector had a delay of about $5 \mathrm{~s}$ in

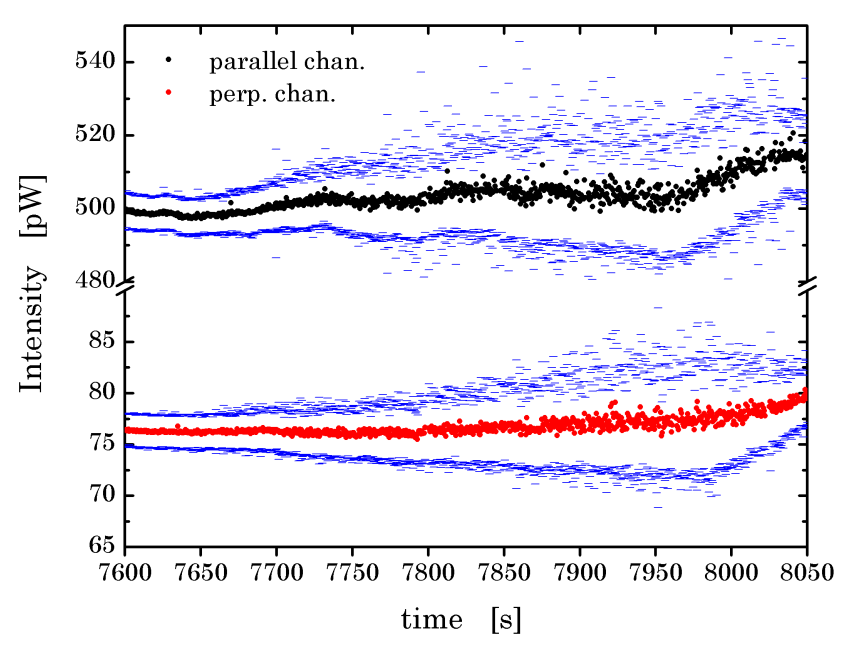

Fig. 4. Real-time signal average values (black and red dots) and their standard deviations (blue dashes) calculated within a 0.5 -s time interval for the Snomax 6th experiment.

comparison to the OPC data. This is a good agreement since the OPC had a time resolution of $5 \mathrm{~s}$. During the activation, the activated fraction determined with the OPC remained between 0.01 and 0.02. No further activation was observed with increasing relative humidity and decreasing temperature. In the IODE data, some large isolated peaks occurred, especially in the parallel channel. First, coincidence events seemed to happen, where simultaneous detection of several particles leads to a much higher intensity and to a miscount of peak numbers. This is mostly the case when concentrations of particles exceed roughly $70 \mathrm{IN}$ per $\mathrm{cm}^{3}$. Secondly, the ice particles are distributed over the entire width of the chamber at the level of the detector. Closer particles thus generate a higher scattered intensity due to the dependence of the scattering intensity on the distance $r$ to the detector (see Eq. 7). A rise in the perpendicular channel intensity also appeared during the activation run: The formation of bigger ice crystals along the experiment and/or a change in the particle shape as a result of varying temperature and relative humidity with respect to ice (Pruppacher and Klett, 1997). Moreover temperature changes in the ZINC chamber can cause a small misalignment between the detector and the chamber, even though they were mechanically coupled but obviously not strong enough. An increase in the depolarization ratio was denoted.

The activation end (shortly before $t=8100 \mathrm{~s}$ ) is well indicated in both the OPC and the IODE detectors. Another way to visualize the activation is to plot the mean values and the standard deviation of the two IODE channels. This is done by taking the average of all the sampled individual points from the real-time signal every $0.5 \mathrm{~s}$. These profiles are illustrated in Fig. 4. 
Table 1. Statistical analysis of the OPC and IODE data during the Snomax 6th activation experiment.

\begin{tabular}{cccc}
\hline & Sum of counts & average [pW] & std. dev. [pW] \\
\hline OPC & 13,777 & - & - \\
$I_{\|}$(ice) & 10,564 & 39.48 & 40.74 \\
$I_{\|}($water $)$ & 16,811 & 24.18 & 15.47 \\
$I_{\perp}$ & 10,564 & 11.83 & 8.29 \\
$\delta_{\perp}$ & - & 0.250 & 0.112 \\
\hline
\end{tabular}

In the mean values, it is hard to see a strong rise, especially in the perpendicular channel. On the contrary, the activation is nicely seen in terms of standard deviations (blue dashes). Their increase results from the individual peaks that enlarge the intensity distribution, making it therefore wider. However, it remains difficult to obtain the exact timing of the start and termination of the activation. Table 1 indicates the values obtained during the Snomax 6th experiment from the IODE detector (intensities and depolarization ratios) and includes the OPC raw data.

The average values of all cases (Table 1) indicate that the large ice particles formed inside the chamber scatter more light than the non-depolarizing particles. These can be either water droplets or other particles that do not depolarize light. It is possible that un-activated aerosol particles that took up water are falsely detected as non-depolarizing particles. The Snomax particles may deliquesce but very likely no efflorescence occurs afterwards in the evaporation section since relative humidities with respect to water are still close to $100 \%$ at the warm temperatures of these experiments. Therefore, the particles exhibit a spherical shape and are large enough to be detected. Some small ice crystals might also exhibit a spherical shape, but even low asphericities can induce strong depolarization signals (Mishchenko and Sassen, 1998). The OPC counted a total amount of 13777 particles during the activation whereas the IODE registered 10564 non-spherical particles during the same time interval. According to previous calibration tests made in the laboratory, the OPC has a detection efficiency between $50 \%$ and $60 \%$ for particles sizes of $0.5 \mu \mathrm{m}$ in diameter, but is very likely to reach $100 \%$ for sizes we expect for our experiment (from 1 to $10 \mu \mathrm{m}$ ). Therefore, the IODE overestimates the total amount of particles, including also coincidence events. This might come from high background signals and/or noise that are counted as particles. The lowpass filter frequency could also be set too high, allowing additional background signals to be falsely recorded.

For comparison with previous model calculations (Nicolet et al., 2007), probability density functions were assembled using only data with $\delta_{\perp} \geqslant 0.04$. This limit cannot be mathematically deduced, but it ensures that the perpendicular light intensity is sufficiently high to be detected by the PMT. The comparison is made between the experimental data and the modeled one for a $2-\mu \mathrm{m}$ diameter particle with an aspect ratio of 1 (Fig. 5).

The average value obtained from the experiment is significantly higher and shows a skewed distribution (right panel). The differences may be caused by the differences in shape and size of the real ice crystals in the ZINC chamber as compared to the idealized shapes used in the simulations. Theoretically, the ice particles should be randomly oriented, given their settling velocity $(\sim 0.4 \mathrm{~m} / \mathrm{s})$ and a diameter range between 1 and $10 \mu \mathrm{m}$ (Hallett, 1987). Moreover, the ice particles were modeled as cylinders while their real shapes should be more complex. Unfortunately, it is not possible to independently determine the size and shape of these parameters and to have clear information about the size and the aspect ratio of these particles. Nevertheless, a similar decrease as calculated can be observed for $\delta_{\perp}>0.4$ with very few occurrences of high depolarization (Nicolet et al., 2007).

\subsection{Israel dust}

In the case of an activation made with Israel dust particles, the concentrations and the size of the aerosol particles were much larger as shown in Fig. 6 (panel b).

The starting point of the activation is clearly identifiable on the activated fraction panel. In that case, the activated fraction constantly increased until reaching the minimum temperature $(237 \mathrm{~K})$ and the maximum humidity with respect to ice (143\%). The same evolution occurred for the parallel and perpendicular peak intensities. This translates into a rise in the particle size in a similar way like in the Snomax activation experiment. On the contrary, the depolarization ratio stayed at the same level, showing that this parameter is no more dependent on the size, as it should be the case for large particle sizes (Nicolet et al., 2007). The activated fractions determined with the OPC and the IODE detectordiffer significantly. Due to the high concentration of aerosols $\left(\sim 2900 \mathrm{~cm}^{-3}\right)$, the detection threshold $\tau$ was difficult to set and was probably too high, which partly explains the few number of ice crystals detected.

\subsection{Saharan dust}

For this experiment, Saharan dust aerosols were injected in the ZINC chamber. The humidity conditions required for this sample were rather higher, peaking at $\mathrm{RH}_{i}=173 \%$ and $\mathrm{RH}_{w}=133 \%$ at temperatures as low as $246.5 \mathrm{~K}$. Figure 7 depicts the same type of activation panels as discussed before.

A "water breakthrough" event as defined in Sect. 4.1 appeared at $t \approx 7500 \mathrm{~s}$. Water droplets which survived the evaporation section appear in the OPC raw data as every bin had a large amount of counts (cyan-green colored bins from channel 50 to 200 in panel b). The activated fraction determined with the OPC rapidly increased to values of about 0.45 and remained constant all along the activation until the temperature was increased. However, this represents activated ice 

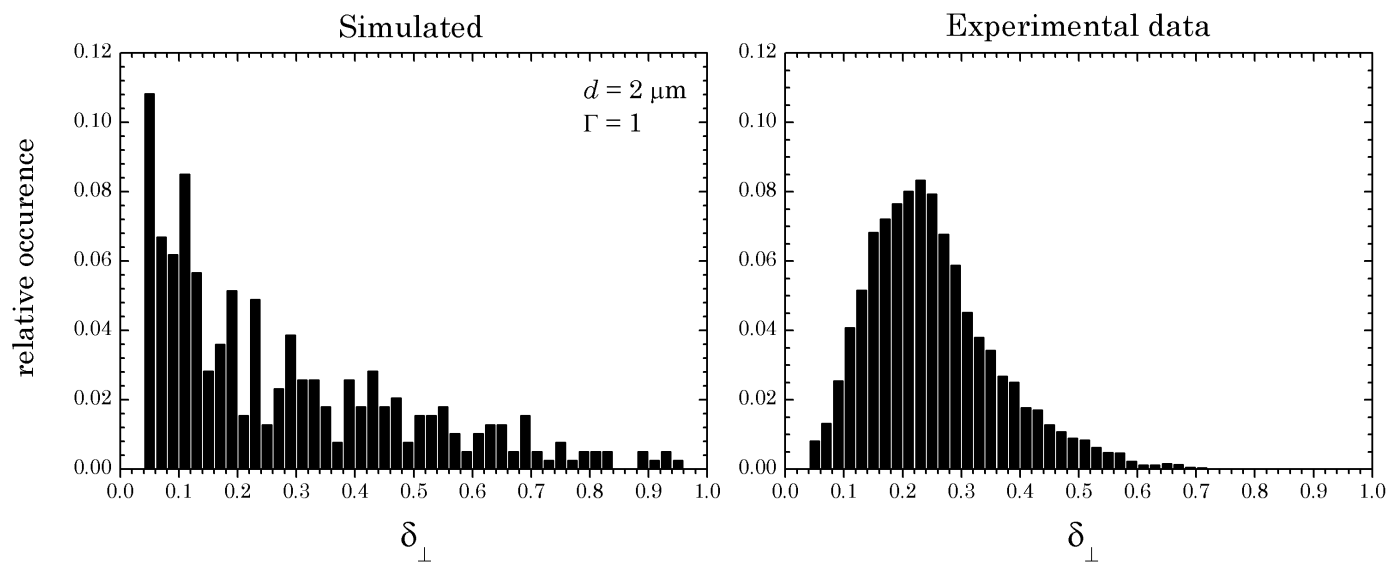

Fig. 5. Simulated occurrence of the depolarization ratio $\delta_{\perp}$ considering a cylindrical ice crystal with an aspect ratio of 1 and a diameter of $2 \mu \mathrm{m}$ (from Nicolet et al., 2007) (left panel) and depolarization ratio $\delta_{\perp}$ obtained during the Snomax 6th activation experiment for the whole IODE dataset (right panel).

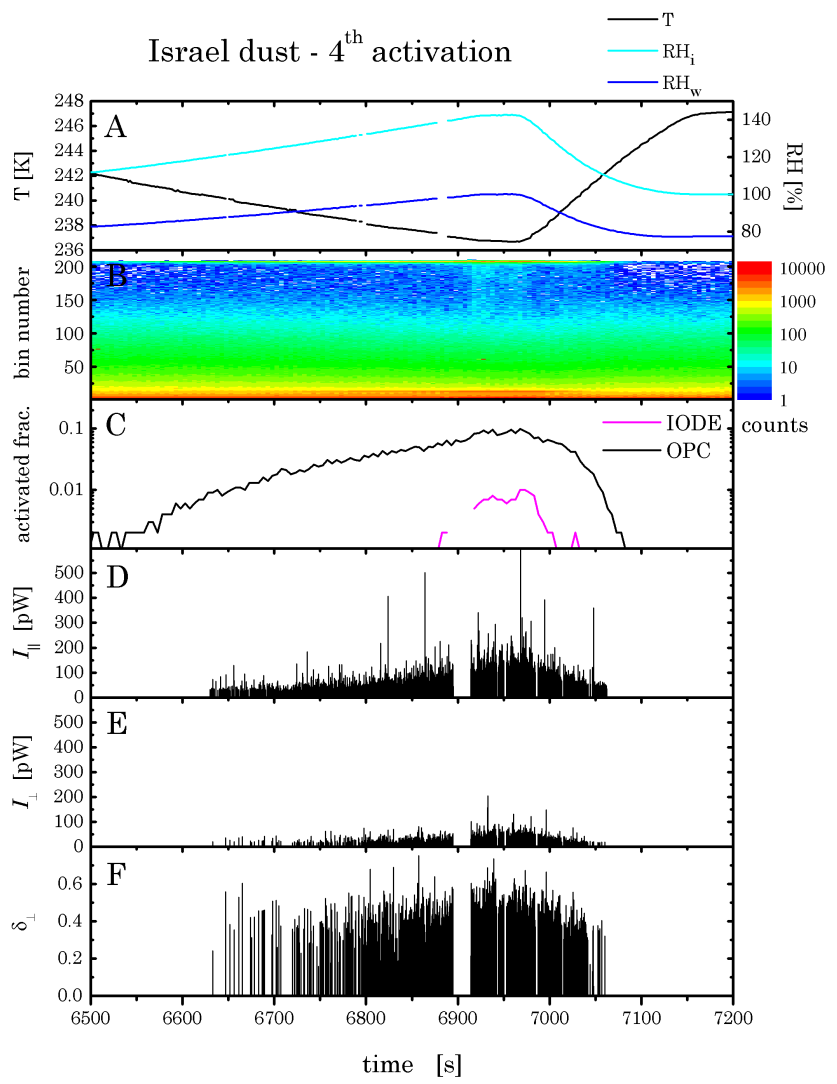

Fig. 6. Scheme of the Israel dust 4 th activation experiment.

particles and water droplets. The real activation profile of ice crystals as measured with the IODE detector did not exceed 0.008 . The number of ice particles detected represented about $1.3 \%$ of all particles recorded during the activation ex-

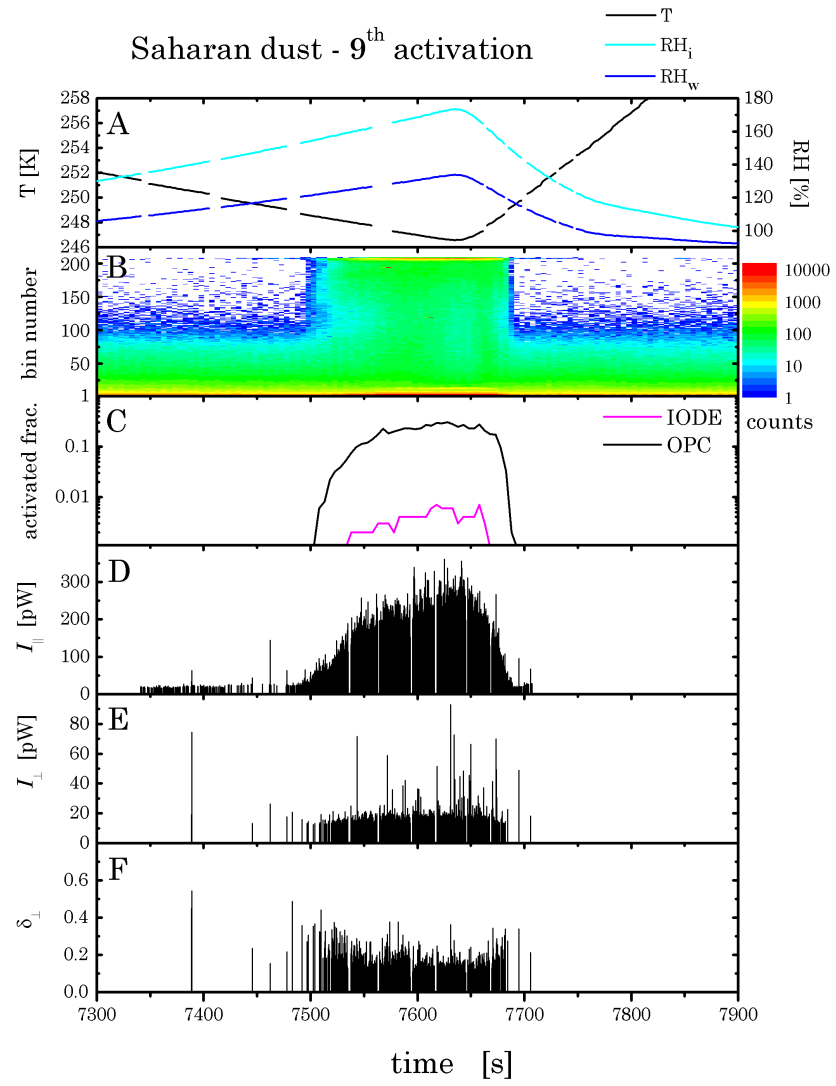

Fig. 7. Scheme of the Saharan dust 9th activation experiment.

periment. In the IODE data, the peak structure was significantly different than the ice activations discussed previously. In the parallel channel, a large increase can be seen whereas the perpendicular peak signals stayed globally constant. The 

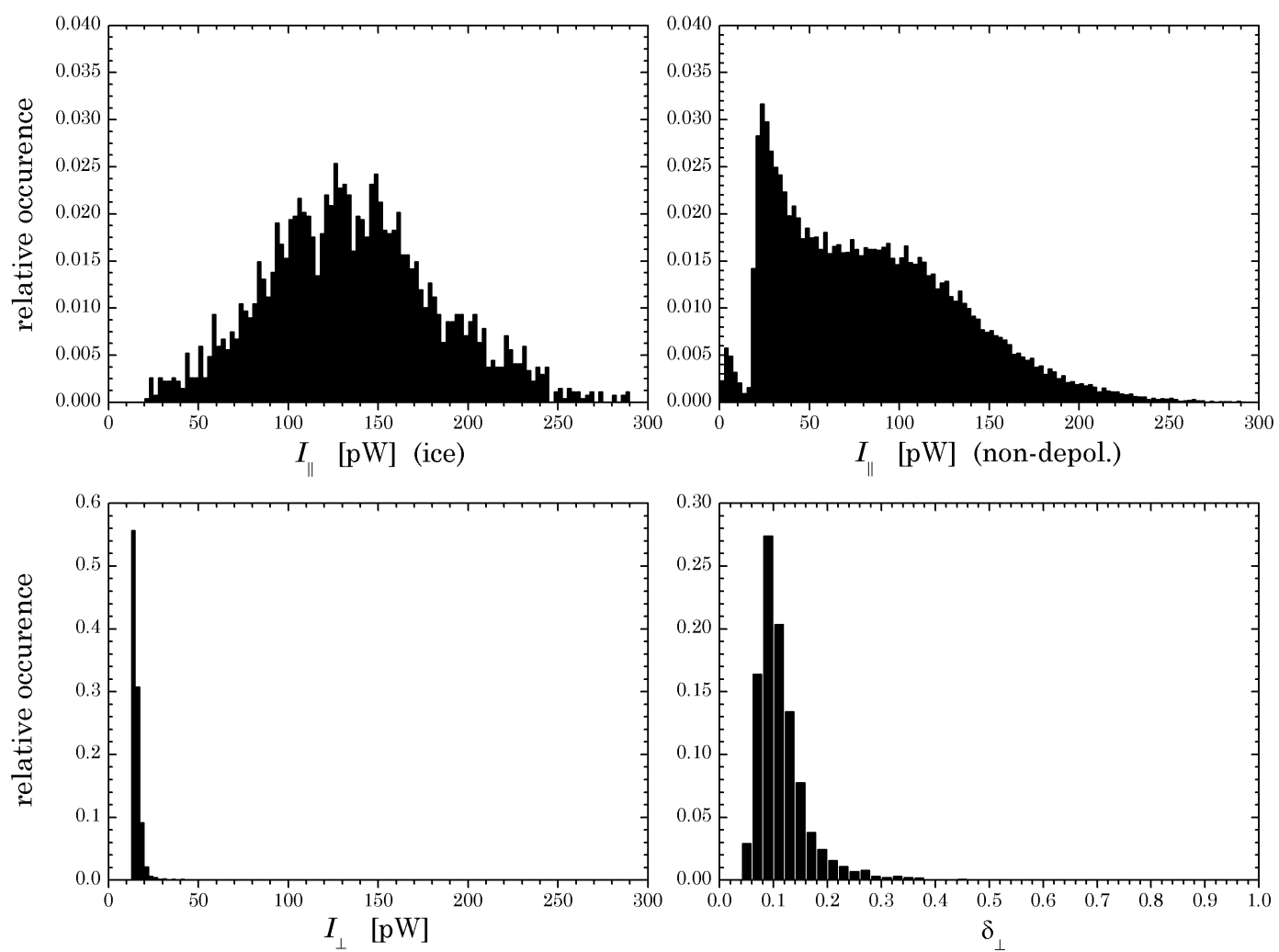

Fig. 8. Histograms of parallel intensities (ice and water particles, upper panels), perpendicular intensities (bottom left panel) and depolarization ratios (bottom right panel) during the Saharan dust 9th activation experiment for the whole IODE dataset.

presence of higher peaks indicated the presence of ice crystals among the water drops. This trend suggests the following explanation: The mixture of water droplets and ice particles caused this overall slight rise in the average signal intensity. It was high enough to detect a high number of low intensity peaks. The statistical analysis of this activation experiment shown in Fig. 8 establishes that the ice crystals detected are a minority of all particles.

Although ice crystals were in the minority, they scattered more light than water drops and exhibit a quasi-normal distribution in the frequency plot. The water droplets scattering intensity showed nearly a double peak structure: We suggest that the first peak is related to single particle detection whereas the second maximum distribution $(95 \mathrm{pW})$ corresponds to coincidence events. Still, the perpendicular channel consisted almost entirely of low intensities. This could indicate a particular shape of the ice crystals that does not generate a strong depolarization effect. In the real-time averaged signal for both channels, the water activation can be clearly identified, too (Fig. 9).

The average and standard deviation signals illustrate a large increase in the parallel channel. The perpendicular one is slightly rising - sufficiently to get all the low peaks as previously discussed - but nothing comparable to the parallel

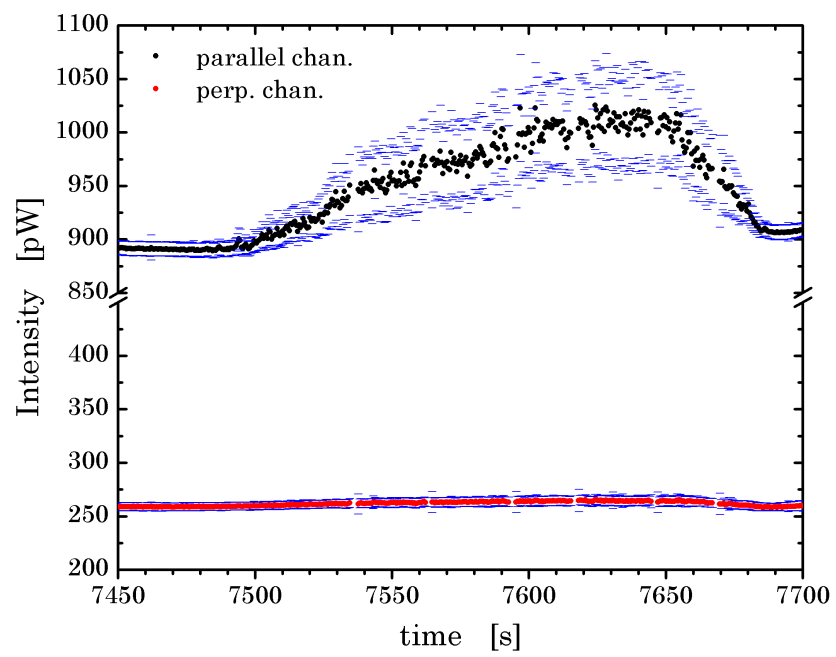

Fig. 9. Real-time signal average values (black and red dots) and their standard deviations (blue dashes) calculated within a 0.5 -s time interval for the Saharan dust 9th activation experiment.

channel. In case of high concentrations, the average-standard deviation method is a good tool to show the presence of water droplets and/or ice crystals. 


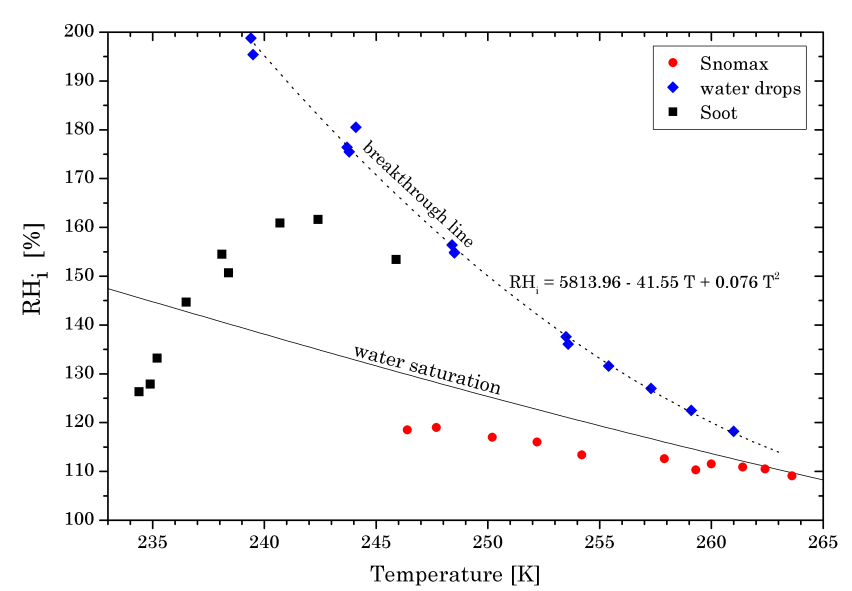

Fig. 10. Critical saturation ratios for Snomax and soot particles obtained from the OPC from ZINC experiments using a 1\%-activated fraction as threshold for the onset of freezing. The plain line shows the water saturation line using the parametrizations from Murphy and Koop (2005) and the dotted line illustrates the breakthrough section where water droplets survive the evaporation section of the ZINC chamber (blue diamonds).

\section{Discussion}

Concerning the water activation experiments discussed previously, they were all recorded in the same way and are plotted in Fig. 10 (blue diamonds) together with Snomax bacteria and soot experiments.

The dotted line defines the "breakthrough" threshold above which water droplets which had formed inside the chamber are surviving the passage through the evaporation section. As all points follow a clear trend, a best fit is given by the dotted line. These water breakthrough events are not dependent on the aerosol type, but are only due to the technical characteristics of the ZINC chamber. It is therefore unique for this chamber. Another example is given in Fig. 11 which depicts the total counts of both the OPC and IODE detectors for a Snomax activation experiment where water droplet breakthrough occurred.

This activation experiment can be divided in two parts: The first one concerns only ice crystals as no water droplets survived the ZINC evaporation section. The ice peak counts from the IODE range between 35\% and $97 \%$ of the total OPC counts, with values mainly around $70 \%$. The most interesting part is the "water breakthrough" occurring at $t=740 \mathrm{~s}$ : From that time, the counts from the OPC and the water peaks determined with the IODE device increased drastically. The temperature was $253.6 \mathrm{~K}$ and we had a relative humidity with respect to ice of $136 \%$ (112.7\% with respect to water). From $t=790 \mathrm{~s}$, the IODE water droplet counts started to stay constant at values of approximately $13 \mathrm{~cm}^{-3}$, showing a saturation effect of the IODE detector whereas the OPC counts continued to climb.

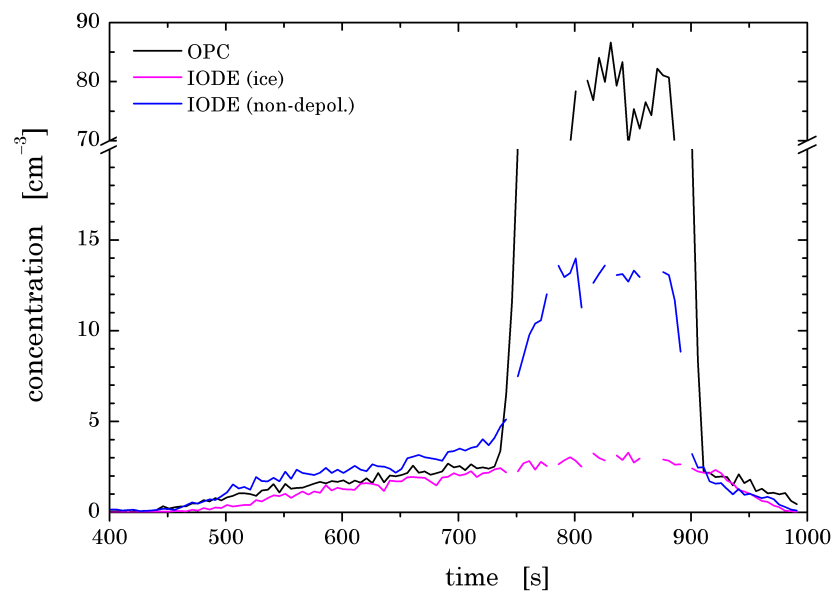

Fig. 11. Optical particle counter counts (black solid line), water peaks (blue line) and ice peaks counts obtained from the IODE detector (magenta solid line) for the Snomax 7th activation experiment and considering an integration time of $5 \mathrm{~s}$.

It is not possible to only rely on the OPC data as it does not distinguish between the water and the ice phase. Consequently, this leads to miscounts of the total particle number and we cannot determine the fraction of ice particles present during these events. However, ice crystals can be observed with the IODE detector even when water breakthrough events occur. Thus, it is possible to show and to follow the formation of ice crystals along these processes. Hence the evaporation section is not necessary anymore for future experiments. An additional information to identify water breakthrough events can be done with a statistical analysis of the depolarization ratios of all ice crystals during a whole activation experiment. This is especially useful in case of high aerosol concentration events.

\section{Conclusions}

A depolarization detector device was designed and realized to detect water droplets and ice particles that can coexist in a diffusion chamber. This system allows the discrimination between the liquid and the solid phase. Ice crystals depolarize the incident polarized light whereas spherical water drops do not. The parallel and the perpendicular component (to the incident light polarization) of the scattering light intensities are measured and the depolarization ratio is determined. Peaks in both channels are counted with a peak detection algorithm. The signal noise is reduced with a lowpass filter. This lowers the standard deviation of the background signal and therefore increases the detection efficiency.

The IODE device was used with the ZINC chamber during the ICIS 2007 workshop in order to demonstrate its potential as a new detector for the ZINC chamber. Three activation 
experiments were discussed. In the first one, ice activation occurred with a low particle concentration (Snomax bacteria). In the second experiment, a higher particle concentration of Israel dust and in the third one of Saharan dust was used. It was possible to identify the onset and termination of each ice activation event with the IODE detector. In the case of ice activation, peak intensities in both parallel and perpendicular channels increased simultaneously. The resulting depolarization ratios showed values ranging from 0.02 up to 0.8 , which demonstrates that single ice particle detection is possible according to theoretical calculations (Nicolet et al., 2007). In the Israel dust experiment, it was also possible to determine the activation onset. Water droplets at very high supersaturations were well determined in the Saharan dust experiment. Even though the particle concentration was very high, causing some saturation problems with the peak algorithm, it was possible to identify the presence of liquid particles by using the 0.5 -s average real-time values together with their standard deviations. This alternative solution works well for high particle concentrations. A clear increase in the parallel channel was seen, whereas the perpendicular channel signal exhibited no significant rise. It was also shown that the ice particles scatter more light than water droplets and have a broader intensity distribution. Thus, they can be discriminated from water droplets. Additionally, it must be mentioned that the detector does not see the aerosol particles as their sizes (reaching up to $500 \mathrm{~nm}$ in diameter roughly) are too small to induce sufficient scattering.

However, some uncertainties might occur during experiments. The alignment of the laser beam passing through the chamber plays a major role in order to miss as few particles as possible. In presence of high particle concentrations, several particles may falsely be counted as a single event. In case of ice crystals, some specific particle orientations do not induce a significant depolarization ratio, causing them to be miscounted as water droplets. A change in the background intensity during experiments can lead to a misinterpretation of the threshold values in the peak algorithm. These variations may be caused by small displacements of the chamber relative to the detector, owing to the walls cooling at different temperatures. This can lead to a miscount of peaks and to apparent background peaks even if there is no activation. For the moment, the readjustments have to be done manually.

The first successful tests demonstrate that the IODE device is a promising tool to detect ice crystals and water droplets simultaneously in cloud diffusion chambers. The main challenge is to be able to lower the detection efficiency as much as possible by reducing the background noise in order to capture also single particle events at low particle concentration. Particle detection in high concentration conditions is a problem: Peak counts have to be as close as possible to the expected known values as saturation might occur, meaning that the IODE detector is not able to count all particles if the concentration is too high. Attaching the IODE detector directly to the ZINC chamber windows (without using a bread- board for the optical elements) can avoid background intensity changes along experiments. Further applications with the IODE device can be made to investigate mixed-phase clouds in airborne experiments where both liquid and solid phases can coexist. We also plan to equip the setup with an additional detector in forward direction to be more sensitive to estimate particle sizes and eventually be able to detect unactivated aerosol particles.

Acknowledgements. The authors thank the reviewers for useful comments and suggestions. They thank the Swiss National Science Foundation for the support of this research under grant 200021-107663/1 and all the AIDA team during the ICIS 2007 workshop.

Edited by: M. Kulmala

\section{References}

Bundke, U., Nilius, B., Jaenicke, R., Wetter, T., Klein, H., and Bingemer, H.: The Fast Ice Nucleus chamber FINCH, Atmos. Res., 90, 180-186, 2008.

Büttner, S.: Streulicht experimente an asphärischen Aerosolpartikeln: depolarization und Vorwärtsstreuverhältnis von Mineralstaub und Eiskristallen: Dissertation FZKA 6989, PhD thesis, Forschungszentrum Karlsruhe, 2004.

Cantrell, W. and Heymsfield, A.: Production of ice in tropospheric clouds - a review, B. Am. Meteorol. Soc., 86, 795-807, 2005.

Forster, P., Ramaswamy, V., Artaxo, P., Bernsten, T., Betts, R., Fahey, D. W., Haywood, J., Lean, J., Lowe, D. C., Myhre, G., Nganga, J., Prinn, R., Raga, G., Schulz, M., and Dorland, R. V.: Climate Change 2007: The Physical Science Basis, Contribution of Working Group I to the Fourth Assessment Report of the Intergovernmental Panel on Climate Change, Cambridge University Press, Cambridge, UK and New York, USA, 2007.

Gőtz, G., Mészáros, E., and Vali, G.: Atmospheric Particles and Nuclei, Akadémiai Kiadó, Budapest, 1991.

Hallett, J.: Faceted snow crystals, J. Opt. Soc. Am. A, 4, 581-588, 1987.

Hirst, E., Kaye, P. H., Greenway, R. S., Field, P., and Johnson, D. W.: Discrimination of micrometer-sized ice and super-cooled droplets in mixed-phase cloud, Atmos. Environ., 35, 315-348, 2001.

Krämer, B., Schwell, M., Hübner, O., Vortisch, H., Leisner, T., Rühl, E., Baumgärtel, H., and Wöste, L.: Homogeneous Ice Nucleation Observed in Single Levitated Micro Droplets, Ber. Bunsenges. Phys. Chem., 100, 1911-1914, 1996.

Lawson, R. P. and Cormack, R. H.: Theoretical design and preliminary tests of two new particle spectrometers for cloud microphysics research, Atmos. Res., 35, 315-348, 1995.

Liou, K. N. and Lahore, H.: Laser sensing of cloud composition: a Backscattered depolarization technique, J. Appl. Meteorol., 13, 257-263, 1974.

Liou, K. N. and Schottland, R. M.: Multiple backscattering and depolarization from water clouds for a pulsed lidar system, J. Atmos. Sci., 28, 772-784, 1971.

Lohmann, U. and Feichter, J.: Global indirect aerosol effects: a review, Atmos. Chem. Phys., 5, 715-737, 2005, http://www.atmos-chem-phys.net/5/715/2005/. 
McFarquhar, G. M., Yang, P., Macke, A., and Baran, A. J.: A new representation of the Single-scattering solar radiative properties for tropical anvils using observed ice crystal size and shape distributions, J. Atmos. Sci., 59, 2458-2478, 2002.

Mishchenko, M. I.: Calculation of the amplitude matrix for a nonspherical particle in a fixed orientation, Appl. Optics, 39, 10261031, 2000.

Mishchenko, M. I. and Hovenier, J. W.: Depolarization of light backscattered by randomly oriented nonspherical particles, Opt. Lett., 20, 1356-1358, 1995.

Mishchenko, M. I. and Sassen, K.: Depolarization of lidar returns by small ice crystals: An application to contrails, Geophys. Res. Lett., 25, 309-312, 1998.

Möhler, O., Stetzer, O., Schaefers, S., Linke, C., Schnaiter, M., Tiede, R., Saathoff, H., Krämer, M., Mangold, A., Budz, P., Zink, P., Schreiner, J., Mauersberger, K., Haag, W., Kärcher, B., and Schurath, U.: Experimental investigation of homogeneous freezing of sulphuric acid particles in the aerosol chamber AIDA, Atmos. Chem. Phys., 3, 211-223, 2003, http://www.atmos-chem-phys.net/3/211/2003/.

Morris, C. E., Georgakopoulos, D. G., and Sands, D. C.: Ice nucleation active bacteria and their potential role in precipitation, $\mathrm{J}$. Phys. IV France, 121, 87-103, 2004.

Murphy, D. and Koop, T.: Review of the vapour pressures of ice and Supercooled water for atmospheric applications, Q. J. Roy. Meteorol. Soc., 6, 2981-2990, 2005.

Nicolet, M., Stetzer, O., and Lohmann, U.: Depolarization ratios of singles ice particles assuming finite circular cylinders, Appl. Optics, 46, 4465-4476, 2007.

Pruppacher, H. R. and Klett, J. D.: Microphysics of Clouds and Precipitation, Kluwer Acad., Norwell, Mass., 1997.
Rogers, D. C.: Development of a continuous flow thermal gradient diffusion chamber for ice nucleation studies, Atmos. Res., 22, 149-181, 1988.

Rogers, D. C.: Measurements of natural ice nuclei with a continuous flow diffusion chamber, Atmos. Res., 29, 209-228, 1993.

Rogers, D. C., DeMott, P. J., Kreidenweis, S. M., and Chen, Y.: A Continuous-flow diffusion chamber for airborne measurements of ice nuclei, J. Atmos. Oceanic Technol., 18, 725-741, 2001.

Sassen, K.: Lidar cloud research, Rev. Laser Eng., 23, 148-153, 1995.

Schnaiter, M., Gimmler, M., Llamas, I., Linke, C., Jäger, C., and Mutschke, H.: Strong spectral dependence of light absorption by organic carbon particles formed by propane combustion, Atmos. Chem. Phys., 6, 2981-2990, 2006, http://www.atmos-chem-phys.net/6/2981/2006/.

Spurny, K.: Atmospheric condensation nuclei P. J. Coulier 1875 and J. Aitken 1880 (historical review), Aerosol. Sci. Technol., 32, 243-248, 2000.

Stetzer, O., Baschek, B., Lüönd, F., and Lohmann, U.: The Zurich Ice Nucleation Chamber (ZINC) - A new instrument to investigate atmospheric ice formation, Aerosol. Sci. Technol., 42, 64$74,2008$.

Szyrmer, W. and Zawadzki, I.: Biogenic and anthropogenic sources of ice-forming nuclei: A review, B. Am. Meteorol. Soc., 78(2), 209-228, 1997.

Vali, G.: Atmospheric ice nucleation - A review, J. Rech. Atmos., 19, 105-115, 1985.

van de Hulst, H. C.: light Scattering by Small Particles, Wiley, NewYork, 1957.

Wood, S. E., Baker, M. B., and Swanson, B. D.: Instrument for studies of homogeneous and heterogeneous ice nucleation in freefalling Supercooled water droplets, Rev. Sci. Instrum., 73, 39883996, 2002. 